\title{
Comparação da aptidão física de idosos praticantes e não praticantes do método Pilates
}

\author{
Daniel Vicentini de Oliveira*, Geisa Luiza Mendes Arantes Alves", Gabriel Lucas Morais Freire ***, \\ Maura Fernandes Franco"***, Mateus Dias Antunes ${ }^{* * * * *}$, José Roberto Andrade do Nascimento Júnior"**, \\ Rogéria Vicentini de Oliveira*, Sônia Maria Marques Gomes Bertolini*
}

\section{Resumo}

Este estudo teve o objetivo de comparar a aptidão física de idosos praticantes e não praticantes do método Pilates. Pesquisa transversal, a qual participaram 120 idosos da cidade de Maringá-PR, sendo 60 praticantes do método Pilates em estúdios e academias, e 60 idosos frequentadores das academias da terceira idade (ATI). Como instrumentos foram utilizados um questionário sociodemográfico e o a Senior Fitness Test. A análise dos dados foi conduzida por meio dos testes de Kolmogorov-Smirnov, Qui-quadrado de Pearson $\left(X^{2}\right)$, Mann-Whitney e coeficiente de Spearman $(p<0,05)$. Os resultados evidenciaram maior proporção de idosos praticantes de Pilates que tomam medicamentos regularmente e maior proporção de idosos frequentadores das ATI que relataram a ausência de doença do coração e osteoporose. Além disso, os idosos frequentadores das ATI apresentaram melhor aptidão física do que os idosos praticantes de Pilates. Concluiu-se que para os idosos avaliados, a prática de atividade de atividade física nas ATI parece ser mais eficiente para a aptidão física dos idosos do que a prática do Pilates. Ainda, os idosos frequentadores das ATI reportaram melhor condição de saúde do que os idosos praticantes de Pilates.

Palavras chave: Atividade motora; Exercício; Envelhecimento; Capacidade física.

\section{Introdução}

O envelhecimento humano é tido como um dos principais fenômenos sociais do século XXI (BEARD et al., 2017) e consiste na degeneração progressiva dos sistemas corporais (CORDEIRO et al., 2014). Afeta a capacidade de funcionamento do corpo e, assim, associa-se à redução da mobilidade e independência dos idosos (LOPES; SANTOS, 2015;

* Departamento de Pós-Graduação em Promoção da Saúde, Centro Universitário de Maringá (Unicesumar) - Maringá (PR), Brasil

** Departamento de Educação Física, Unicesumar - Maringá (PR), Brasil

*** Departamento de Pós-Graduação em Educação Física, Universidade Federal do Vale do São Francisco (UNIVASF) - Petrolina (PE), Brasil

**** Departamento de Pós-Graduação em Gerontologia, Faculdade de Ciências Médicas, Universidade Estadual de Campinas (UNICAMP) - Campinas (SP), Brasil

****** Departamento de Pós-Graduação em Ciências da Reabilitação, Universidade de São Paulo (USP) - São Paulo (SP), Brasil

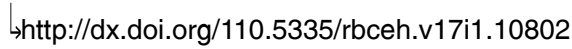


VANCAMPFORT; STUBBS; KOYANAGI, 2017).

Essas alterações prejudicam negativamente os domínios biopsicossociais e podem por em risco a qualidade de vida do idoso (SPOSITO; NERI; YASSUDA, 2016). Restrições nos níveis de saúde física e mental, prejuízos na realização de atividades de vida diária (AVD) e nos níveis funcionalidade e autonomia (LOPES; SANTOS, 2015; SPOSITO; NERI; YASSUDA, 2016) são bastante comuns nesse processo e tendem a aumentar o risco de sedentarismo nessa população (PAULA et al., 2015).

O sedentarismo vem sendo alvo de pesquisas e sofrendo importante pressão do avanço tecnológico nas últimas décadas (LOPES; SANTOS, 2015; PAULA et $a l .$, 2015; VANCAMPFORT; STUBBS; KOYANAGI, 2017) visto que é um dos principais fatores de risco aos agravos à saúde e um dos mais prevalentes na população mundial (SIQUEIRA et al., 2009). No Brasil, a inatividade física entre pessoas de baixa renda e sua prevalência na população em geral aumentou de $41,1 \%$ para $52,0 \%$ entre os anos de 2002 e 2007 (KNUTH et al., 2010). A literatura aponta ainda que ela tende a aumentar com o avanço da idade e que pessoas acima de 60 anos são as mais acometidas (HALLAL, 2012; RIBEIRO et al., 2015).

Por sua vez, relacionada à saúde, a aptidão física contempla a flexibilidade, a força e resistência muscular, a composição corporal e a capacidade cardiorrespiratória. Estes componentes estão relacionados ao estado físico, psicológico e social, nos aspectos de promoção e prevenção da saúde, assim como redução dos riscos de doenças, como também pela maior disposição para as AVD (RIBEIRO et al., 2015).

Neste sentido, a prática de exercício físico se destaca combatendo o sedentarismo, e contribuindo significativamente para a manutenção da aptidão física relacionada à saúde do idoso, seja na sua vertente da saúde como nas capacidades funcionais (FERREIRA et al., 2012; SILVA et al., 2016; WARBURTON; BREDIN, 2017). Estudos demonstram que atividades que tem como objetivo melhorar a força e resistência muscular, a flexibilidade e o condicionamento aeróbico oferecem efeitos positivos à capacidade funcional de idosos (BAZZANELLA; PICCOLI; QUEVEDO, 2015; CEDERHOLM; CRUZ-JENTOFT; MAGGI, 2013; JACINTO; BUZZACHERA; AGUIAR, 2016).

Dentre as diversas modalidades de exercícios, o método Pilates tem sido bastante aconselhado à população idosa, e parece ser bem aceito entre eles (DELLA TORRE et al., 2017; PARIKH; ARORA, 2016). O método foi desenvolvido há mais de um século por Joseph Pilates e, mesmo com suas múltiplas ramificações, nota-se que o trabalho com o método se pauta na grande variedade de movimentos aliados a uma respiração adequada. Esse processo faz com que o exercício seja executado de forma controlada e fluída, alongando músculos encurtados e fortalecendo músculos fracos, de forma equilibrada e resulta na melhora da postural corporal, redução dos níveis de dor, de 
stress e consequentemente na melhora da funcionalidade (BERTOLI; BIDUSKI; FREITAS, 2017; DELLA TORRE et al., 2017; PARIKH; ARORA, 2016).

Este estudo justifica-se pelo fato de a literatura, apesar de ser bastante enfática a cerca dos benefícios da atividade física para aos idosos (ENGERS et al., 2016; NASCIMENTO et al., 2018; RODRIGUES et al., 2010), ela ainda é pouco conclusiva em relação a esses benefícios entre os praticantes e não praticantes do método Pilates. Neste contexto, este estudo buscou comparar a aptidão física de idosos praticantes e não praticantes do método Pilates.

\section{Materiais e métodos}

\section{Participantes}

A amostra deste estudo foi escolhida de forma intencional e por conveniência, e foi composta por 120 idosos, sendo 60 praticantes do método Pilates nos estúdios e academias selecionadas para a pesquisa, e 60 idosos frequentadores das academias da terceira idade (ATI), selecionadas para a pesquisa. Foram incluídos idosos de ambos os sexos, praticantes exclusivamente do método Pilates, ou usuário da ATI. Idosos que utilizavam acessórios para marcha (bengala, andador, dentre outros), assim como aqueles submetidos a cirurgias de grande porte que podiam limitar a realização do teste, foram excluídos. Os idosos usuários das ATI, que praticavam outras modalidades de exercícios físicos que não fosse o método Pilates, foram excluídos.

\section{Instrumentos}

Para avaliar o perfil sociodemográfico, de saúde e de prática do método Pilates, foi utilizado um questionário estruturado pelos próprios autores com questões referentes à: idade, faixa etária (60 a $69 ; 70$ a $79 ; 80$ ou mais), estado conjugal (com ou sem companheiro - a), escolaridade (não estudou; fundamental incompleto; fundamental completo; médio completo; superior), renda mensal em salários mínimos (SM) (um a dois SM; dois a três salários mínimos; mais de três salários mínimos), aposentadoria (sim; não), percepção da saúde (boa; regular; ruim), comparação da saúde com pessoas da mesma idade (pior; igual; melhor), uso de medicamentos (sim; não), histórico de queda e/ou quase queda no último semestre (sim; não), frequência semanal da prática de Pilates (duas; três; quatro vezes ou mais), tempo de prática do Pilates (três meses a um ano; um a três anos; mais de três anos), presença e/ou histórico de doenças crônicas não transmissíveis.

Para avaliação da aptidão física do idoso, foi utilizada parte da bateria de testes de Rikli e Jones (2002), a Senior Fitness Test. A mesma envolve os testes de flexão de cotovelo; levantar e sentar da cadeira; levantar, andar 2,44 metros e voltar a sentar; alcançar a frente; alcançar atrás; e teste de caminhada de seis minutos. O teste de caminhada de seis minutos foi substituído pelo teste de marcha estacionária de dois minutos (RIKILI; JONES, 1999). 


\section{Procedimentos}

Estudo observacional e transversal, aprovado pelo comitê de ética e pesquisa do Centro Universitário Metropolitano de Maringá por meio do parecer número 3.096.769/2018.

Uma listagem das academias e estúdios de Pilates do município de Maringá foi adquirida por meio de busca eletrônica na internet, na mesma constavam 59 estúdios e academias. Dez delas foram sorteadas. Após entrar em contato via telefone com cada uma delas e explicar o objetivo e procedimentos da pesquisa, oito das 10 empresas aceitaram participar e assinaram a declaração de concordância dos serviços envolvidos e/ou de instituição coparticipante. Não foi necessário seguir o mesmo procedimento com os idosos de exercícios nas ATI, por ser local aberto ao público. Quatro ATI foram utilizadas como local de coleta de dados.

Cada responsável pelos locais de pesquisa (academias e estúdios de Pilates) agendaram com os idosos para a coleta dos dados. Nas ATI as coletas foram feitas em dias e horários diversos, de acordo com a disponibilidade do pesquisador. Cada coleta individual durou em média 15 minutos tendo sido o idoso devidamente esclarecido sobre os procedimentos da pesquisa assinando assim o termo de consentimento livre e esclarecido.

\section{Análise dos dados}

A análise dos dados foi realizada por meio do Software SPSS 22.0, mediante uma abordagem de estatística descritiva e inferencial. Foi utilizado frequência e percentual como medidas descritivas para as variáveis categóricas. Para as variáveis numéricas, inicialmente foi verificada a normalidade dos dados por meio do teste Kolmogorov-Smirnov. Como os dados não apresentaram distribuição normal foi utilizada mediana (Md) e quartis (Q1; Q3) para a caracterização dos resultados. $\mathrm{O}$ teste de Qui-quadrado de Pearson $\left(\mathrm{X}^{2}\right)$ foi utilizado para se observar às possíveis diferenças nas proporções das variáveis sociodemográficas e das condições de saúde de acordo com a prática de pilates foi efetuada por meio do teste "U" de Mann-Whitney. A correlação entre os testes de aptidão física foi verificada por meio do coeficiente de Spearman. Foi adotada a significância de $\mathrm{p}<0,05$.

\section{Resultados}

Ao comparar as proporções das variáveis sócio demográficas de acordo com a prática de Pilates (Tabela 1), verificou-se diferença significativa entre os grupos apenas na faixa etária $(\mathrm{p}=0,005)$, indicando que existe maior proporção de idosos não praticantes de Pilates na faixa etária de 60 a 70 anos (83,3\%). Já ao comparar as proporções das variáveis relacionadas às condições de saúde em função da prática de Pilates (Tabela 1), foi encontrada diferença significativa entre os grupos apenas no uso de medicamentos $(p=0,031)$, evidenciando que existe maior proporção de idosos praticantes de Pilates que tomam medicamentos regularmente. 
Tabela 1: Comparação das proporções das variáveis sócio demográficas dos idosos praticantes e não praticantes do método Pilates do município de Maringá, PR

\begin{tabular}{|c|c|c|c|c|}
\hline \multirow{3}{*}{ Variáveis } & \multicolumn{2}{|c|}{ Prática de Pilates } & \multirow{3}{*}{$X^{2}$} & \multirow{3}{*}{$\mathrm{p}$} \\
\hline & $\operatorname{Sim}(n=60)$ & Não $(n=60)$ & & \\
\hline & $f(\%)$ & $f(\%)$ & & \\
\hline \multicolumn{5}{|l|}{ Sexo } \\
\hline Feminino & $47(78,3)$ & $45(75,0)$ & 0.100 & 0960 \\
\hline Masculino & $13(21,7)$ & $15(25,0)$ & 0,186 & 0,666 \\
\hline \multicolumn{5}{|l|}{ Faixa etária } \\
\hline 60 a 70 anos & $35(58,3)$ & $50(83,3)$ & & \\
\hline 71 a 79 anos & $17(28,3)$ & $7(11,7)$ & 7,876 & $0,005^{*}$ \\
\hline 80 anos ou mais & $8(13,3)$ & $3(5,0)$ & & \\
\hline \multicolumn{5}{|l|}{ Estado conjugal } \\
\hline Com companheiro & $36(60,0)$ & $39(65,0)$ & & $57 ?$ \\
\hline Sem companheiro & $24(40,0)$ & $21(35,0)$ & 0,320 & $0,5 / 2$ \\
\hline \multicolumn{5}{|l|}{ Cor } \\
\hline Branca & $54(90,0)$ & $48(80,0)$ & & \\
\hline Preta & $1(1,7)$ & $4(6,7)$ & 1,640 & 0,203 \\
\hline Amarela & $5(8,3)$ & $8(13,3)$ & & \\
\hline \multicolumn{5}{|l|}{ Escolaridade } \\
\hline Analfabeto & $0(0,0)$ & $1(1,7)$ & & \\
\hline Fund. Incompleto & $4(6,7)$ & $8(13,3)$ & & \\
\hline Fund. Completo & $7(11,7)$ & $9(15,0)$ & 2,028 & 0,154 \\
\hline Médio completo & $21(35,0)$ & $17(28,3)$ & & \\
\hline Superior & $28(46,6)$ & $25(41,7)$ & & \\
\hline \multicolumn{5}{|l|}{ Aposentadoria ${ }^{a}$} \\
\hline Sim & $39(65,0)$ & $37(62,7)$ & 0067 & 0795 \\
\hline Não & $21(35,0)$ & $22(37,3)$ & 0,001 & 0,195 \\
\hline \multicolumn{5}{|l|}{ Renda mensala } \\
\hline 1 a 2 SM & $1(1,8)$ & $10(19,2)$ & & \\
\hline 2,1 a $3 \mathrm{SM}$ & $22(40,0)$ & $14(26,9)$ & 2,760 & 0,097 \\
\hline Mais de $3 \mathrm{SM}$ & $32(58,2)$ & $28(53,9)$ & & \\
\hline Percepção de saúde & & & & \\
\hline Boa & $42(70,0)$ & $40(66,7)$ & & \\
\hline Regular & $14(23,3)$ & $19(31,7)$ & 0,026 & 0,871 \\
\hline Ruim & $4(6,7)$ & $1(1,7)$ & & \\
\hline \multicolumn{5}{|l|}{ Percepção de saúde comparada a outros idosos } \\
\hline Pior & $3(5,0)$ & $3(5,0)$ & & \\
\hline Igual & $22(36,7)$ & $29(48,3)$ & 1,159 & 0,282 \\
\hline Melhor & $35(58,3)$ & $28(46,7)$ & & \\
\hline \multicolumn{5}{|l|}{ Medicamentos } \\
\hline Sim & $54(90,0)$ & $45(75,0)$ & & \\
\hline Não & $6(10,0)$ & $15(25,0)$ & $4,6 / 5$ & $0,031 \wedge$ \\
\hline \multicolumn{5}{|l|}{ Quantidade de medicamentos } \\
\hline Nenhum & $6(10,0)$ & $11(18,3)$ & & \\
\hline 1 a 2 & $21(35,0)$ & $26(43,3)$ & 3,699 & 0,054 \\
\hline Mais de 2 & $33(55,0)$ & $23(38,3)$ & & \\
\hline \multicolumn{5}{|l|}{ Quedas } \\
\hline Sim & $12(20,0)$ & $7(11,7)$ & & 0211 \\
\hline Não & $48(80,0)$ & $53(88,3)$ & 1,563 & 0,211 \\
\hline \multicolumn{5}{|l|}{ Quase quedas } \\
\hline Sim & $9(15,0)$ & $6(10,0)$ & 0686 & 0.408 \\
\hline Não & $51(85,0)$ & $54(90,0)$ & 0,000 & 0,400 \\
\hline
\end{tabular}

*Associação significativa $-\mathrm{p}<0,05$ : Teste de Qui-quadrado para proporções. ${ }^{a}$ Variáveis com casos ausentes. SM: salário (s) mínimo (s). 
Ao comparar as proporções das doenças relatadas pelos idosos de acordo com a prática de Pilates (Tabela 2), houve diferença significativa apenas na doença do coração $(\mathrm{p}=0,040)$ e na osteoporose ( $\mathrm{p}=0,007)$, indicando maior proporção de idosos não praticantes de Pilates que relataram a ausência de ambas as doenças.

Tabela 2: Comparação das proporções das doenças relatadas pelos idosos praticantes e não praticantes do Método Pilates do município de Maringá, PR

\begin{tabular}{|c|c|c|c|}
\hline \multirow{3}{*}{ Variáveis } & \multicolumn{2}{|c|}{ Prática de Pilates } & \multirow{3}{*}{$X^{2}$} \\
\hline & $\underset{(n=60)}{\operatorname{Sim}}$ & $\begin{array}{c}\text { Não } \\
(\mathrm{n}=60)\end{array}$ & \\
\hline & $f(\%)$ & $f(\%)$ & \\
\hline \multicolumn{4}{|c|}{ Doença do coração } \\
\hline Sim & $10(16,7)$ & $3(5,0)$ & \multirow{2}{*}{4,227} \\
\hline Não & $50(83,3)$ & $57(95,0)$ & \\
\hline \multicolumn{4}{|l|}{ Hipertensão } \\
\hline Sim & $30(50,0)$ & $20(33,3)$ & \multirow{2}{*}{3,429} \\
\hline Não & $30(50,0)$ & $40(66,7)$ & \\
\hline \multicolumn{4}{|l|}{ AVE } \\
\hline Sim & $2(3,3)$ & $3(5,0)$ & \multirow{2}{*}{0,209} \\
\hline Não & $58(96,7)$ & $57(95,0)$ & \\
\hline \multicolumn{4}{|l|}{ Diabetes } \\
\hline Sim & $14(23,3)$ & $7(11,7)$ & \multirow{2}{*}{2,828} \\
\hline Não & $46(76,7)$ & $53(88,3)$ & \\
\hline \multicolumn{4}{|l|}{ Câncer } \\
\hline Sim & $4(6,7)$ & $2(3,3)$ & \multirow{2}{*}{0,702} \\
\hline Não & $56(93,3)$ & $58(96,7)$ & \\
\hline \multicolumn{4}{|l|}{ Osteoartrite } \\
\hline Sim & $23(38,3)$ & $15(25,0)$ & \multirow{2}{*}{2,465} \\
\hline Não & $37(61,7)$ & $45(75,0)$ & \\
\hline \multicolumn{4}{|c|}{ Doenças do pulmão } \\
\hline Sim & $5(8,3)$ & $3(5,0)$ & \multirow[t]{2}{*}{0,536} \\
\hline Não & $55(91,7)$ & $57(95,0)$ & \\
\hline \multicolumn{4}{|l|}{ Depressão } \\
\hline Sim & $9(15,0)$ & $9(15,0)$ & \multirow[t]{2}{*}{0,000} \\
\hline Não & $51(85,0)$ & $51(85,0)$ & \\
\hline \multicolumn{4}{|l|}{ Osteoporose } \\
\hline Sim & $22(36,7)$ & $9(15,0)$ & \multirow{2}{*}{7,350} \\
\hline Não & $38(63,3)$ & $51(85,0)$ & \\
\hline
\end{tabular}

* Associação significativa - p < 0,05: Teste de Qui-quadrado para proporções. AVE: acidente vascular encefálico.
Ao comparar as proporções das variáveis relacionadas à aptidão física dos idosos de acordo com a prática de Pilates (Tabela 3), foi encontrada diferença significativa apenas na classificação da marcha estacionária $(p=0,038)$ e na força muscular de membros superiores $(p=0,001)$, indicando maior proporção de idosos não praticantes de Pilates com nível bom em ambos os testes de aptidão física.

Tabela 3: Comparação das proporções das variáveis relacionadas à aptidão física dos idosos praticantes e não praticantes do método Pilates do município de Maringá, PR

\begin{tabular}{|c|c|c|c|c|}
\hline \multirow{3}{*}{ Variáveis } & \multicolumn{2}{|c|}{ Prática de Pilates } & \multirow{3}{*}{$X^{2}$} & \multirow{3}{*}{$\mathrm{p}$} \\
\hline & \multicolumn{2}{|c|}{$\operatorname{Sim}(n=60)$ Não $(n=60)$} & & \\
\hline & $f(\%)$ & $f(\%)$ & & \\
\hline \multicolumn{5}{|c|}{ Marcha estacionária } \\
\hline Fraco & $1(1,7)$ & $1(1,7)$ & & \\
\hline Regular & $8(13,3)$ & $0(0,0)$ & 4,288 & $0,038^{*}$ \\
\hline Bom & $51(85,0)$ & $59(98,3)$ & & \\
\hline
\end{tabular}

Força de membros inferiores

\begin{tabular}{l|r|r|r|r} 
Fraco & $21(35,0)$ & $10(16,7)$ & & \\
Regular & $15(25,0)$ & $7(11,7)$ & 10,235 & $0,001^{*}$ \\
Bom & $24(40,0)$ & $43(71,7)$ & & \\
\hline
\end{tabular}

Força de membros superiores

\begin{tabular}{l|r|r|r|r} 
Fraco & $1(1,7)$ & $1(1,7)$ & & 1 \\
Regular & $2(3,3)$ & $2(3,3)$ & 0,000 & 1,000 \\
Bom & $57(95,0)$ & $57(95,0)$ & & \\
\hline
\end{tabular}

Flexibilidade de membros superiores

\begin{tabular}{l|l|l|l|l}
\hline Fraco & $36(60,0)$ & $28(46,7)$ & & \\
Regular & $11(18,3)$ & $21(35,0)$ & 0,478 & 0,489 \\
Bom & $13(21,7)$ & $11(18,3)$ & & \\
\hline
\end{tabular}

Flexibilidade de membros inferiores

\begin{tabular}{l|l|l|l|l}
\hline Fraco & $22(36,7)$ & $13(21,7)$ & & \\
Regular & $15(25,0)$ & $31(51,7)$ & 0,054 & 0,817 \\
Bom & $23(38,3)$ & $16(26,7)$ & & \\
\hline
\end{tabular}

Mobilidade

\begin{tabular}{l|l|l|l|l}
\hline Fraco & $20(33,3)$ & $14(23,3)$ & & \\
Regular & $18(30,0)$ & $25(41,7)$ & 0,325 & 0,569 \\
Bom & $22(36,7)$ & $21(35,0)$ & & \\
\hline
\end{tabular}

* Associação significativa - p < 0,05: Teste de Qui-quadrado para proporções. 
Ao comparar os resultados brutos dos testes de aptidão física dos idosos em função da prática de Pilates (Tabela 4), verificou-se diferença significativa entre os grupos nos testes de marcha estacionária $(p=0,001)$, flexão de coto- velo ( $\mathrm{p}=0,004)$ e caminhar 2,44 metros e sentar $(p=0,002)$. Estes resultados evidenciam que os idosos não praticantes de Pilates apresentaram melhor aptidão física nos três testes.

Tabela 4: Comparação da aptidão física dos idosos praticantes e não praticantes de Pilates da cidade de Maringá-PR

\begin{tabular}{|c|c|c|c|}
\hline \multirow{3}{*}{ Variáveis } & \multicolumn{2}{|c|}{ Prática de Pilates } & \multirow{2}{*}{$p$} \\
\hline & $\operatorname{Sim}(n=60)$ & Não $(n=60)$ & \\
\hline & Md (Q1; Q3) & Md (Q1; Q3) & \\
\hline Marcha estacionária (Passos) & $91,5(80,0 ; 102,0)$ & $108,0(100,3 ; 118,0)$ & $0,001^{*}$ \\
\hline Sentar e levantar (Repetições) & $16,0(12,0 ; 20,8)$ & $19,5(16,0 ; 22,0)$ & 0,289 \\
\hline Flexão de cotovelo (Repetições) & $27,0(23,0 ; 33,0)$ & $29,5(24,0 ; 33,0)$ & $0,004^{*}$ \\
\hline Alcançar atrás das costas $(\mathrm{cm})$ & $-6,5(-14,8 ; 0,0)$ & $-0,5(-10,8 ; 0,0)$ & 0,174 \\
\hline Alcançar a frente $(\mathrm{cm})$ & $0,0(-8,5 ; 8,8)$ & $0,0(0,0 ; 4,5)$ & 0,861 \\
\hline Caminhar $2,44 \mathrm{~m}$ e sentar (segundos) & $5,9(5,1 ; 7,5)$ & $5,2(4,7 ; 6,1)$ & $0,002^{*}$ \\
\hline
\end{tabular}

* Diferença significativa: $\mathrm{p}<0,05$ - Teste "U" de Mann-Whitney.

Ao analisar a correlação entre os testes de aptidão física dos idosos praticantes de Pilates (Tabela 5), verificou-se as seguintes correlações significativas $(\mathrm{p}<0,05)$ : marcha estacionária com flexão de cotovelo $(\mathrm{r}=61)$, sentar e levantar $(\mathrm{r}=0,69)$, alcançar a frente $(\mathrm{r}=0,36)$ e caminhar 2,44 metros ( $\mathrm{r}=-0,60)$; flexão de cotovelo com sentar e levantar $(r=0,72)$, alcançar a frente $(r=0,27)$ e caminhar 2,44 metros $(r=-0,53)$; sentar e levantar com alcanças atrás das costas $(r=0,28)$, alcançar a frente $(\mathrm{r}=0,52)$ e caminhar 2,44 metros $(\mathrm{r}=-0,68)$; alcançar atrás das costas com alcançar a frente $(\mathrm{r}=0,49)$ e caminhar 2,44 metros ( $r=-0,35)$; e alcançar a frente com caminhar 2,44 metros $(\mathrm{r}=-0,39)$.

Tabela 5: Correlação entre os testes de aptidão física dos idosos praticantes e não praticantes de do método Pilates

\begin{tabular}{|c|c|c|c|c|c|c|}
\hline $\begin{array}{l}\text { Praticantes } \\
\text { Não praticantes }\end{array}$ & 1 & 2 & 3 & 4 & 5 & 6 \\
\hline 1. Marcha estacionária & - & $0,61^{*}$ & $0,69^{*}$ & 0,25 & $0,36^{*}$ & $-0,60^{\star}$ \\
\hline 2. Flexão de cotovelo & $0,38^{*}$ & - & $0,72^{*}$ & 0,08 & $0,27^{*}$ & $-0,53^{\star}$ \\
\hline 3. Sentar e levantar & $0,47^{*}$ & $0,49^{*}$ & - & $0,28^{*}$ & $0,52^{*}$ & $-0,68^{\star}$ \\
\hline 4. Alcançar atrás das costas & 0,19 & $-0,11$ & 0,23 & - & $0,49^{*}$ & $-0,35^{\star}$ \\
\hline 5. Alcançar a frente & 0,18 & 0,10 & $0,27^{*}$ & $0,40^{*}$ & - & $-0,39 *$ \\
\hline 6. Caminhar $2,44 \mathrm{~m}$ e sentar & $-0,43^{*}$ & $-0,33^{*}$ & $-0,37^{*}$ & $-0,18$ & $-0,14$ & - \\
\hline
\end{tabular}

*Correlação significativa $(\mathrm{p}<0,05)$ - Correlação de Spearman. 
Para os idosos não praticantes de Pilates, foram encontradas as seguintes correlações significativas $(\mathrm{p}<0,05)$ : marcha estacionária com flexão de cotovelo ( $\mathrm{r}=38$ ), sentar e levantar $(\mathrm{r}=0,47)$ e caminhar 2,44 metros $(r=-0,43)$; flexão de cotovelo com sentar e levantar $(r=0,49)$ e caminhar 2,44 metros $(r=-0,33)$; sentar e levantar com alcançar a frente $(r=0,27)$ e caminhar 2,44 metros $(r=-0,37)$; alcançar atrás das costas com alcançar a frente $(r=0,40)$. Estes achados indicam que existe uma relação linear mais forte entre os testes de aptidão física para os idosos praticantes de Pilates.

\section{Discussão}

O objetivo da presente investigação foi analisar a aptidão física de idosos praticantes e não praticantes do método Pilates. Os principais achados desta investigação foram a maior evidencia de idosos que não pratica Pilates com idade de 60 a 70 anos; uma maior parte da amostra que pratica Pilates toma algum tipo de medicamento, enquanto os idosos que não praticam Pilates não relatam doenças. Em relação aos testes de aptidão física os idosos que não praticavam Pilates apresentaram um bom nível nos três testes.

Um achado interessante da presente investigação, foi ao analisar a correlação entre os testes de aptidão física dos idosos praticantes de Pilates. Estes achados indicam que existe uma relação linear mais forte entre os testes de aptidão física para os idosos praticantes de Pilates. Esses achados corroboram com Oliveira et al. (2013), que encontrou melhoras significa- tivas no grupo que realizou a intervenção com o Pilates quando comparado ao grupo controle. Um agregado de autores assegura que o Pilates está associado a melhora de estabilidade, equilíbrio, flexibilidade, coordenação e força fazendo com que os idosos sintam menos dificuldades para realizar os movimentos.

Em mais uma análise, agora relacionado a comparação dos testes de aptidão física. Pode se observar diferença entre os grupos em especial nos testes de marcha estacionaria, flexão de cotovelo e caminhar, 2,44m. Esses achados demostram que os idosos não praticantes de Pilates apresentam melhor aptidão física nesses testes. Esses achados discordam do estudo de Rosa et al. (2013) que comparou grupo praticantes de Pilates com grupo de sedentários. Os autores conseguiram observar que o grupo de Pilates apresentou melhora significativa nos testes de força e marcha. Uma provável explicação é não sabermos o tipo de atividade que os não praticantes de Pilates realiza, podendo estas está relacionada ao melhor desempenho nos testes. Com isso, a prática de exercício físico deve ser estimulada para essa população devido à importância dessa variável para realizações das atividades de vida diárias, prevenção de sarcopenia e fragilidade (OLIVEIRA et al., 2019; RODRIGUES et al., 2010).

Ao comparar as proporções de doenças de acordo com a prática de Pilates é possível observar diferença em doenças do coração e osteoporose. Indicando que idosos não praticantes da modalidade relatam a ausência de ambas as doenças. Uma provável explicação é o fato 
do Pilates ser uma atividade de baixa intensidade e não gerar impacto possibilitando assim a pratica desses indivíduos (TORQUATO et al., 2016).

Esses achados são de suma importância para corroborar com a lacuna da literatura sobre a temática (OLIVEIRAet al., 2013). Embora esse estudo apresente algumas limitações que devem ser consideradas. A primeira é a falta de descrição de atividades realizadas pelos idosos sem ser o Pilates, bem como a não descrição detalhada do método de Pilates praticado pelos mesmos que pode ser feita por aparelhos ou exercícios no solo sem acessórios (OLIVEIRA et al., 2013; OLIVEIRA et al., 2019). Segundo é o local do estudo, por não ser uma capital, a amostra acaba sendo pequena podendo assim prejudicar os resultados. Desta forma, há limitações para realizar comparações dos resultados. Outra limitação se refere ao fato de selecionar os indivíduos de forma não-probabilística, o que também limita a interpretação dos resultados. Com isso, sugere-se pesquisas relacionados a estas temáticas para consolidar de vez este feixe na literatura, bem como melhorar a adesão de idosos em programa de atividades. Estudos sobre modalidade de exercício físico para idosos são de suma importância para auxiliar os profissionais na forma de intervenção com esta população, bem como prescrição da intensidade do treinamento.

\section{Conclusão}

Concluiu-se que idosos não praticantes do método Pilates, na cidade avaliada, possuem, de forma geral, melhor aptidão física do que os idosos praticantes do método. Ainda, os idosos não praticantes do método reportaram melhor condição de saúde do que os idosos praticantes.

\section{Comparison of physical fitness of older adults practicing and non practitioners Pilates method}

\section{Abstract}

This study aimed to analyze the physical fitness of practicing and non-Pilates older adults. A cross-section research, realized with of 120 older adults from the city of Maringá-PR, 60 of which were Pilates practitioners in studios and academies, and 60 older adults attending the Fitness Zone (FZ). As instruments were used a sociodemographic questionnaire and the Senior Fitness Test. Data analysis was performed using the Kolmogorov-Smirnov, Pearson's Chi-square $\left(X^{2}\right)$, Mann-Whitney and Spearman's coefficients $(p<0.05)$. The results showed a higher proportion of older adults Pilates practitioners taking medications regularly and a higher proportion of elderly attending FZ who reported the absence of heart disease and osteoporosis. In addition, the elderly attending the $\mathrm{FZ}$ presented better physical fitness than the older adults Pilates practitioners. It was concluded that for the older adults evaluated, the practice of physical activity activity in the FZ seems to be more efficient for the physical fitness of the older adults than the Pilates practice. In addition, older FZ patients reported better health status than older Pilates practitioners.

Keywords: Motor activity; Exercise; Aging; Physical capacity. 


\section{Referências}

BAZZANELLA, N.A.L.; PICCOLI, J.C.J.; QUEVEDO, D.M. Qualidade de vida percebida e atividade física: um estudo em idosas acima de 80 anos participantes de um programa municipal de saúde da terceira idade na serra gaúcha, RS. Estudos Interdisciplinares Sobre o Envelhecimento, Porto Alegre, v. 20, n. 1, p. 249-270, 2015.

BEARD, J.R.; CARVALHO, I. A. DE; SUMI, Y.; OFFICER, A; THIYAGARAJAN, J. A. Healthy ageing: moving forward. Bulletin of the World Health Organization, v. 95, n. 11, p. 730-730, 2017. Doi: 10.2471/ BLT.17.203745

BERTOLI, J.; BIDUSKI, G.M.; FREITAS, C.L.R. Six weeks of Mat Pilates training are enough to improve functional capacity in elderly women. Journal of Bodywork and Movement Therapies, v. 21, n. 4, p. 10031008, 2017. Doi: 10.1016/j.jbmt.2016.12.001

CEDERHOLM, T.; CRUZ-JENTOFT, A.J.; MAGGI, S. Sarcopenia and fragility fractures. European Journal of Physical and Rehabilitation Medicine, v. 49, n. 1, p. 111-117, 2013.

CORDEIRO, J.; DEL CASTILLO, B. L.; de FREITAS, C. S.; GONÇALVES, M. P. Efeitos da atividade física na memória declarativa, capacidade funcional e qualidade de vida em idosos. Revista Brasileira de Geriatria e Gerontologia, v. 17, n. 3, p. 541-552, 2014. Doi: 10.1590/1809-9823.2014.13006

DELLA TORRE, A.P.; GEREVINI, H. H. P.; DA SILVA, Y. C. B.; CHAMMA, B. M.; MOUSSA, L. A influência do Método Pilates no público idoso. Revista Pesquisa e Ação, v. 3, n. 2, p. 58-72, 2017.

ENGERS, P.B.; ROMBALDI, A. J.; PORTELLA, E. G.; DA SILVA, M. C. Efeitos da prática do método Pilates em idosos: uma revisão sistemática. Revista Brasileira de Reumatologia, v. 56, n. 4, p. 352-365, 2016. Doi: 10.1016/j.rbr.2015.11.003
FERREIRA, O.G.L.; MACIEL, S. C.; COSTA, S. M. G.; SILVA, A. O.; MOREIRA, M. A. S. P. Envelhecimento ativo e sua relação com a independência funcional. Texto \& Contexto-Enfermagem, v. 21, n. 3, p. 513-518, 2012. Doi: 10.1590/S010407072012000300004

HALLAL, P. C.; ANDERSEN, L. B.; BULL, F. C.; GUTHOLD, R.; HASKELL, W.; EKELUND, U. Global physical activity levels: surveillance progress, pitfalls, and prospects. The Lancet, v. 380, n. 9838, p. 247-257, 2012. Doi: 10.1016/S01406736(12)60646-1

JACINTO, J.L.; BUZZACHERA, C.F.; AGUIAR, A.F. Efeitos da caminhada em ritmo prescrito e autosselecionado sobre a capacidade funcional de mulheres idosas. Journal of Health Sciences, v. 18, n. 4, p. 25763, 2016. Doi: 10.17921/2447-8938.2016v18 n4p257-63

KNUTH, A.G.; BACCHIERI, G.; VICTORA, C. G.; HALLAL, P. C. Changes in physical activity among Brazilian adults over a 5-year period. Journal of Epidemiology \& Community Health, v. 64, n. 7, p. 591-595, 2010. Doi: $10.1136 /$ jech.2009.088526

LOPES, G. L.; SANTOS, M. I. P. O. Funcionalidade de idosos cadastrados em uma unidade da Estratégia Saúde da Família segundo categorias da Classificação Internacional de Funcionalidade. Revista brasileira de geriatria e gerontologia, v. 18 , n. 1, p. 71-83, 2015. Doi: 10.1590/18099823.2015.14013.

NASCIMENTO, M. M. RIOS, P. M. B.; SILVA, C. N.; RODRIGUES, C. M.; DE OLIVEIRA, E. C. F. Efeitos da prática regular do método Pilates sobre a percepção da qualidade de vida de mulheres sexagenárias e septuagenárias. Revista Brasileira de Qualidade de Vida, v.10, n.2, p.1-16, 2018. Doi: 10.3895/rbqv.v10n2.7804 
PAULA, A.F.M. de; FALSARELLA, G. R.; D'ELBOUX, M. J.; GUARIENTO, M. E. Perfis de funcionalidade e relação com óbito em idosos assistidos em Serviço Ambulatorial de Geriatria/Functionality profiles related to mortality in elders assisted in a Geriatric Outpatient Service. Cadernos Brasileiros de Terapia Ocupacional, v. 23, n. 1, 2015. Doi: 10.4322/0104-4931.ctoAO470

PARIKH, C. M.; ARORA, M. Role of Pilates in rehabilitation: A literature review. International Journal of Therapies and Rehabilitation Research, v. 5, n. 4, p. 77-83, 2016. Doi: 10.5455/ijtrr.000000148

RIBEIRO, D. K. M. N.; LENARDT, M. H.; MICHEL, T.; SETOGUCHI, L. S.; GRDEN C. R. B.; OLIVEIRA, E. S. DE. Fatores contributivos para a independência funcional de idosos longevos. Revista da Escola de Enfermagem da USP, v. 49, n. 1, p. 89-96, 2015. Doi: 10.1590/S0080623420150000100012

RIKLI, R. E.; JONES, C. J. Measuring functional fitness of older adults. Journal Active Aging, v. 1, n. 1, p. 24-30, 2002.

RIKLI, R. E.; JONES, C. J. Development and validation of a functional fitness test for community-residing older adults. Journal of Aging and Physical Activity, v. 7, n. 2, p. 129-161, 1999. Doi: 10.1123/ japa.7.2.129

RODRIGUES, B. G. S.; CADER, S. A.; TORRES, N. V. O. B.; OLIVEIRA, E. M. DE; DANTAS, E. H. M. Autonomia funcional de idosas praticantes de Pilates. Fisioterapia e Pesquisa, v. 17, n. 4, p. 300-305, 2010. Doi: 10.1590/S1809-29502010000400003

ROSA, K.B. LIPOSCKI, D.; WALTRICK, T.; SLONGO, A. Qualidade de vida e avaliação funcional em idosos praticantes de Pilates e idosos sedentários. Revista Interdisciplinar de Estudos em saúde, v. 2, n. 1, p. 18-28, 2013. Doi: 10.33362/ries.v2i1.40
SILVA, J. F.; NASCIMENTO JÚNIOR, J. R. A. DO; ARAÚJO, A. P. S. DE; DE OLIVEIRA, D. V. Análise comparativa da qualidade de vida de idosas praticantes de exercícios físicos em centros esportivos e nas academias da terceira idade. Revista Brasileira de Ciências do Envelhecimento Humano, v. 13, n. 3, 2016. Doi: 10.5335/rbceh.v13i3.5726

SIQUEIRA, F.C.V.; NAHAS, M. V.; FACCHINI, L. A.; PICCINI, R. X.; TOMASI, E.; THUMÉ, E.; SILVEIRA, D. S. DA; HALLAL, P. C. Atividade física em profissionais de saúde do Sul e Nordeste do Brasil. Cadernos de Saúde Pública, v. 25, p. 1917-1928, 2009. Doi: 10.1590/S0102311X2009000900006

SPOSITO, G.; NERI, A.L.; YASSUDA, M.S. Atividades avançadas de vida diária (AAVDs) e o desempenho cognitivo em idosos residentes na comunidade: Dados do Estudo FIBRA Polo UNICAMP. Revista Brasileira de Geriatria e Gerontologia, v. 19 , n. 1, p. 7-20, 2016. Doi: 10.1590/18099823.2016.15044

OLIVEIRA, D. V.; SOUZA, A. M. DE, FERREIRA, A. G.; ANTUNES, M. D.; LOVATO, N. S.; NASCIMENTO JÚNIOR, J. R. A. DO. Evaluating the muscular strength, functional capacity, and risk of sarcopenia in elderly women who practice Mat Pilates. Fisioterapia em Movimento, v. 32, 2019. 10.1590/1980-5918.032.ao22

OLIVEIRA, L.C. et al. Efeitos do método Pilates na autonomia funcional de idosas fisicamente ativas. Association Posturologie Internationale, v. 11, n. 53, p. 395, 2013.

TORQUATO, E.; GERAGE, A.; MEURER, S.; BORGES, R.; SILVA, S.; BENEDETTI, T. Comparação do nível de atividade física medido por acelerômetro e questionário IPAQ em idosos. Revista Brasileira de Atividade Física \& Saúde, v. 21, n. 2, p. 144-153, 2016. Doi: 10.12820/rbafs.v. $21 \mathrm{n} 2 \mathrm{p} 144-153$ 
VANCAMPFORT, D.; STUBBS, B.; KOYANAGI, A. Physical chronic conditions, multimorbidity and sedentary behavior amongst middle-aged and older adults in six low-and middle-income countries. International Journal of Behavioral Nutrition and Physical Activity, v. 14, n. 1, p. 147, 2017. Doi: 10.1186/s12966-017-0602-Z

WARBURTON, D. E. R.; BREDIN, S. S. D. Health benefits of physical activity: a systematic review of current systematic reviews. Current Opinion in Cardiology, v. 32 , n. 5, p. 541-556, 2017. Doi: 10.1097/ HCO.0000000000000437

Avenida Guedner, 160. Maringá, Paraná, Brasil Fone: (44) 99942-8575

E-mail: d.vicentini@hotmail.com 